\title{
Gaia: organisation and challenges for the data processing
}

\author{
F. Mignard ${ }^{1}$, C. Bailer-Jones ${ }^{2}$, U. Bastian ${ }^{3}$, R. Drimmel ${ }^{4}$, L. Eyer ${ }^{5}$,

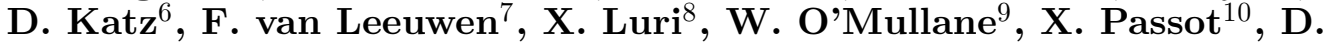 \\ Pourbaix $^{11}$ and T. Prusti ${ }^{12}$ \\ ${ }^{1}$ Observatoire de la Côte d'Azur, Nice, France \\ email: francois.mignard@oca.eu \\ ${ }^{2}$ Max Planck Institut für Astronomie, Heidelberg, Germany \\ ${ }^{3}$ Astronomisches Recheninstitut (ARI), Heidelberg, Germany \\ ${ }^{4}$ Osservatorio Astronomico di Torino, Torino, Italy \\ ${ }^{5}$ Observatoire de l'Université de Genève, Sauverny, Switzerland \\ ${ }^{6}$ Observatoire de Paris-Meudon, Meudon, France \\ ${ }^{7}$ Institute of Astronomy, Cambridge, England \\ ${ }^{8}$ University of Barcelona, Barcelona, Spain \\ ${ }^{9}$ European Space Astronomy Centre, Madrid, Spain \\ ${ }^{10}$ Centre National d'Etudes Spatiales (CNES), Toulouse, France \\ ${ }^{11}$ Université Libre de Bruxelles, Brussels, Belgium \\ ${ }^{12}$ European Space Agency, ESTEC, Noordwijk, The Netherlands
}

\begin{abstract}
Gaia is an ambitious space astrometry mission of ESA with a main objective to map the sky in astrometry and photometry down to a magnitude 20 by the end of the next decade. While the mission is built and operated by ESA and an industrial consortium, the data processing is entrusted to a consortium formed by the scientific community, which was formed in 2006 and formally selected by ESA one year later. The satellite will downlink around $100 \mathrm{~TB}$ of raw telemetry data over a mission duration of 5 years from which a very complex iterative processing will lead to the final science output: astrometry with a final accuracy of a few tens of microarcseconds, epoch photometry in wide and narrow bands, radial velocity and spectra for the stars brighter than 17 mag. We discuss the general principles and main difficulties of this very large data processing and present the organization of the European Consortium responsible for its design and implementation.
\end{abstract}

Keywords. astrometry, instrumentation: photometers, techniques: spectroscopic, methods: data analysis

\section{Introduction}

The ESA mission Gaia is a powerful astronomical space project dedicated to high precision astrometry, photometry and spectroscopy. The details of the science objectives together with the principles of the measurements are given in a companion paper (Lindegren et al., 2008) in this proceeding and are not repeated. Gaia will survey the whole sky and detect any sufficiently point-like source brighter than the 20th magnitude. The observations are carried out with a scanning instrument mapping the instantaneous field of view (composed of two disconnected areas on the sky) onto a single mosaic of CCD very precisely mounted on the focal plane. Therefore each detectable source is observed during its motion on the focal plane brought about by the spin of the satellite. On the 
average a source will transit about 80 times during the five years of the observing mission, leading to about 1000 individual observations per object. They will be more or less regularly distributed over about $\sim 40$ epochs with about one cluster of $\sim 25$ individual observations every 6 weeks. These observations consist primarily of a $1 \mathrm{D}$ accurate determination of the image location at the transit time on a frame rigidly attached to the payload together with an estimate of the source brightness. The number of sources potentially observable is of the order of one billion, primarily stars. In addition there will be something like $\sim 5 \times 10^{5}$ QSOs and $\sim 3 \times 10^{5}$ solar system objects.

\section{Challenges with the data processing}

Scientifically valuable information gathered by Gaia during its lifetime will be encased in the nearly continuous data stream resulting from the collection of photons in the approximately 100 on-board CCDs in the astrometric, photometric and spectroscopic fields of Gaia. However in its original telemetry format the data are totally unintelligible to scientists, not only because it is squeezed into packets by the numerical coding but, more significantly, because of the way Gaia scans the sky. With a $1 \mathrm{D}$ measurement at each source transits, Gaia picks up tiny fragments of the astrometric parameters of the one billion observable sources in the sky. To translate this information into positional and physical parameters, as expected by scientists, a large and complex data analysis must be implemented. How huge and how complex it is, is briefly addressed here.

\subsection{Volume of data}

Data collected by the Gaia detectors will be transmitted by the spacecraft from its position around L2 at a few Mbps for at least five years. Over its lifetime the satellite will deliver to the ground station an uncompressed raw data set of approximately $100 \mathrm{~TB}$, primarily composed of CCD counts collected during the transit of celestial sources on the detector. This will make up the raw data. However the data processing will generate numerous intermediate data to produce the scientifically meaningful data products by 2020. Given these intermediate steps, the need to store the provisional solutions and different backups, it is estimated that the actual size of the Gaia related data will be in the range of a $\mathrm{PB}$ ( $10^{15}$ Bytes), still a fairly large volume by today's standard, not yet matched by any other space mission, although ground based experiments in particle physics will produce data volume one or two orders of magnitude larger. It is enough to say that to store the text of all the books published each year a storage space of about $1 \mathrm{~TB}$ is sufficient which could be readily available on anyone's desk. An orderly access to this data is another question. For the astronomers accustomed to working with the CDS on-line data bases, all the catalogues stored in the VizieR service make less than 1 TB in disk space, while the Aladin sky atlas with the images is just above 5 TB. Despite this apparently limited volume, the administration and maintenance of these services require several permanent staff. Therefore, the volume alone is not a major issue, while efficient access to the relevant piece of data is a serious one.

The core of the final data products distributed to the scientific community will be much smaller. Scaling from Hipparcos with $10^{5}$ sources with all the results on a few CDs, this should amount for Gaia to something around $20 \mathrm{~TB}$ of data. This depends very much on whether raw or nearly raw data are included in the delivery. If one considers just the final data of astrometry, photometry and spectroscopy (not the spectra but their exploitation in terms of radial velocity and astrophysical diagnostic), all the Gaia data expected for a standard user can be estimated by considering a data base with $10^{9}$ entries, each of them comprising less than 100 fields. The astrometric data will need many significant 
digits (or bytes), but most of the other fields will require a limited number of digits or bits. A volume of 1-3 TB should then be sufficient for the main Gaia product, not including data produced during the intermediate steps of the data processing. These data will certainly be made available with calibration data to allow specific reprocessing with improved models by users.

\subsection{Computational complexity}

There is no all-purpose definition of what a complex computation is. It could be complex because of the number of elementary operations it requires (time complexity), or because of the data management (interface complexity), or because of the size of the core memory needed to perform some parts (space complexity). Gaia can be considered complex at least on two counts:

- It implies a very large resource of CPU, or more or less equivalently, the number of basic arithmetical operations is very large by today's standards, and will remain large in the coming ten years.

- The data are widely interconnected with access sometimes to subsets arranged in chronological order (observations of a certain type in a certain time interval) or by sources (all the observations of a subset of sources). This extends to the access of intermediate data or calibration files.

What is at stake can be easily grasped if we consider that there will be about $10^{9}$ individual sources to be processed. Just spending one second on each of them to get the calibrations, spacecraft attitude, astrometric parameters, multi-color photometry and spectra would amount to 30 years of computation. Obviously the overall system must be very efficient to have a chance to produce the scientific results no longer than in three years after the end of the operations.

As to the actual computation size, it is not easy to assess with reliability within at least one order of magnitude as shown already in 2004 by M. Perryman (Perryman, 2004). However we have now a better view with scalable experiments on simulated data. The core iterative processing needs (referred as to AGIS) have been evaluated by Lammers (Lammers, 2006) to $4 \times 10^{19}$ FLOPs for the last cycle, when all the observation material is available. Allowing for the intermediate cycles this yields $2 \times 10^{20}$ FLOPs for the astrometric processing once the image parameters task and source matching are completed. This latter processing has also been evaluated from realistic simulated telemetry and should not be larger than $4 \times 10^{18}$ FLOPs for the full telemetry volume. Regarding the other processing (complex sources, photometry, spectroscopy and before all the image updating) the uncertainty remains significant and depends also on how much additional modelling will be required to account for the radiation effect on the CCDs. A conservative estimate indicates that the most demanding task will be the image updating (improvement of the image parameters and initial centroiding once a better spacecraft attitude and calibrations are known). It is estimated to be of the order of $10^{21}$ FLOPs. But with numerous small computations repeated on all the sources instead of a global adjustment requiring a big core memory access, this demanding subsystem could be either distributed or implemented with a parallel architecture.

The number of $10^{21}$ FLOPs happens to be very close to the largest computations undertaken so far, like a search of the largest prime numbers or the location of the non trivial zeros of the Riemann $\zeta$ function. The recent distributed computation for the protein structure (FAH, 2007) is also in the same area in terms of computing size. However these computations are very different from Gaia's, since they are distributed 


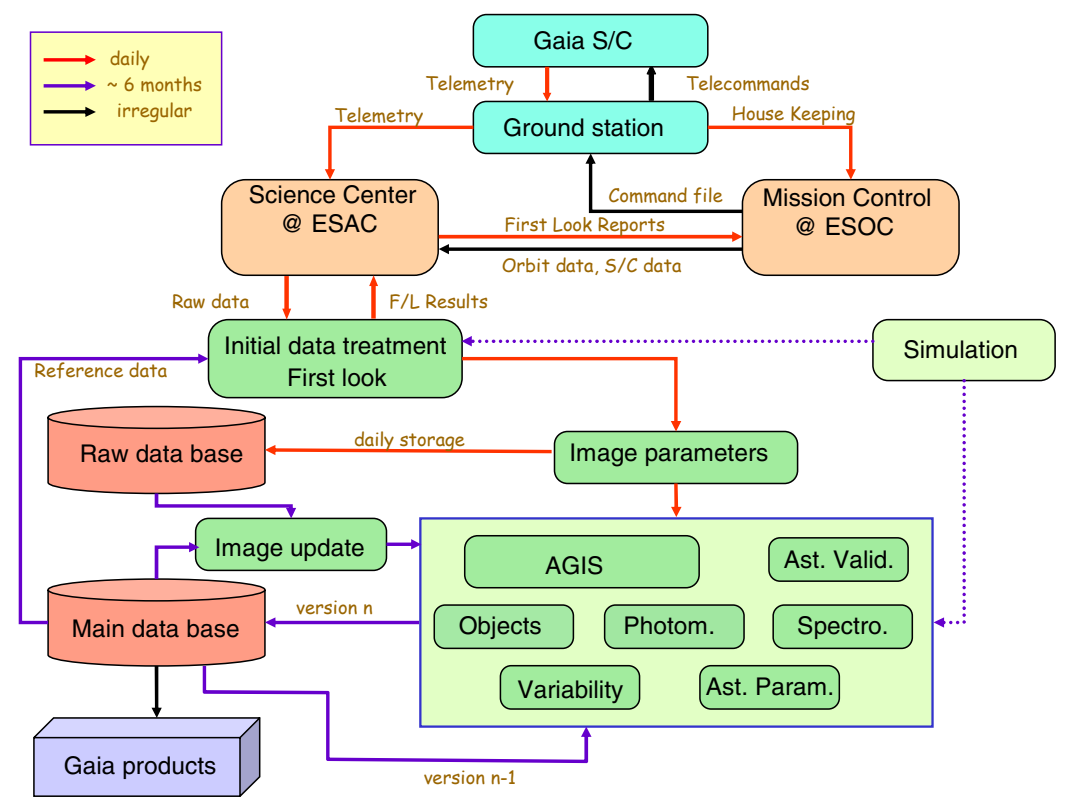

Figure 1. Main structure of the data flow in the processing of the Gaia raw data.

through internet on several thousands processing units of small power (and cheap) and they do not have significant data handling. They are pure computations where each unit is given the task of making a tiny contribution. Most of the Gaia computations cannot be distributed in this way and each computing center will have to access a large amount of data with a significant I/O overhead.

By the time Gaia flies the computing power available to the Gaia Data Processing Centers is expected to be close to $10-20 \mathrm{TFLOP} / \mathrm{s}$ on large clusters. In addition one can also count on the part-time availability of the Barcelona Super Computer (Mare Nostrum) which has a today's power of $20 \mathrm{TFLOP} / \mathrm{s}$. A straight evaluation for Gaia tells that about 2 full years of CPU at $20 \mathrm{TFLOP} / \mathrm{s}$ will be sufficient to process the data. This is still daunting but tractable, provided the estimate is not wrong by two orders of magnitude.

\section{Overview of the data processing}

The Gaia data processing is based on an iterative self-calibrating procedure where the astrometric core solution is the single largest treatment and the cornerstone of the whole processing. These iterations reflect the fact that, as a survey satellite reaches the accuracy and completeness levels never obtained before, it has to be self-calibrated in nearly all aspects of its observations. The most obvious in this respect are the astrometric data: the same data that will ultimately form the astrometric catalogue are also used to reconstruct the scan-phase of the satellite (along-the-scan attitude), which is the reference frame for the astrometric measurements. Similar considerations apply to the spectroscopic and photometric data. Additional complications arise from the fact that these three main iterative solutions are in various ways interlinked and rely on common calibration data.

The overall processing starts with an initial data treatment aiming to obtain a first estimate of the star positions and magnitudes together with an initial attitude, primarily 
based on the on-board crude determination improved on the ground with more accurate (and more computer greedy) algorithms. The next step is the iterative astrometric core solution which provides the calibration data and attitude solution needed for all the other treatments, in addition to the astrometric solution of about 100 million primary sources and the rigid reference system. Once these two steps are performed and their products stored in the central data base, the more specialized tasks are launched with the photometric processing and variability detection, the global analysis of the spectroscopic data and the data treatment for all difficult sources, like planets and multiple stars, that do not fit in the general astrometric or photometric solution of single stars. Two more processing steps close the chain, dealing with the analysis of all types of variable stars and retrieval of their stellar astrophysical parameters like luminosity, temperature or chemical composition. Each step in the processing using earlier data has its own logic regarding the products it will deliver, but that must be seen as part of the validation of the upstream processes. The overall data flow from telemetry to the final products is sketched in Fig. 1. The daily flow follows naturally from the data downlink taking place once per day during the visibility of L2 above the ESA main station in Spain.

The data exchange between the different processing centers (DPCs) takes place via a Main Database (MDB) hosted at a single place. Intermediate processed data from the processing centers flow into the MDB; the processing centers can then extract the data from this Database for further processing. Thus, the Main Database acts as the hub for the Gaia data processing system and must have an efficient and reliable connection with all the DPCs. During operations, the plan is to update this database at regular intervals, typically every six months, corresponding roughly to the period for the satellite to scan the whole celestial sphere. In this way each version of the Main Database is derived from the data in the previous version, supplemented with the processed new observations.

Table 1. Coordination Units of the DPAC and their current leader.

\begin{tabular}{lllll}
\hline CU & Name & Leader & Affiliation & Location \\
\hline CU1 & System Architecture & W. O'Mullane & ESAC & Madrid \\
CU2 & Simulation & X. Luri & UB & Barcelona \\
CU3 & Core processing & U. Bastian & ARI & Heidelberg \\
CU4 & Object processing & D. Pourbaix & ULB & Brussels \\
CU5 & Photometric processing & F. van Leeuwen & IOAC & Cambridge \\
CU6 & Spectroscopic processing & D. Katz & OBSPM & Paris \\
CU7 & Variability processing & L. Eyer & Obs. Geneva & Geneva \\
CU8 & Astrophysical parameters & C. Bailer-Jones & MPIA & Heidelberg \\
\hline
\end{tabular}

\section{Organization of the scientific community}

To cope with the processing challenge, the scientific community, together with ESA, has set up a data processing ground segment comprising a single processing system (no overall duplication) which will deliver the intermediate and final mission science products. Since mission selection, the underlying principles of the data processing have been developed by the Gaia scientific community and individual pieces were successfully tested on small or intermediate size simulations. During this phase one has attempted to identify the critical elements of this processing (size, iterative procedures, instrument 
calibration, data exchange, human and financial resources, computing power) and assess the risks inherent to an endeavor of that size, unprecedented in astronomy.

Based on these preparatory activities the community has joined forces into a dedicated consortium: the Data Processing and Analysis Consortium (the DPAC). In short, the DPAC is a European collaboration including the ESA Gaia Science Operations Centre (SOC) and a broad, international science community of over 320 individuals, distributed over more than 15 countries, including six large Data Processing Centers (DPCs, Table 2). The Consortium has carefully estimated the effort required and has united in a single organization the material, financial and human resources, plus appropriate expertise, needed to conduct this processing to its completion in around 2020. In 2006 the DPAC has proposed to ESA a complete data processing system capable of handling the full size and complexity of the real data within the tight schedule of the mission. The details of this system, its expected performances, funding, organization and management are described in a document submitted to ESA as a Response to its Announcement of Opportunity for the Gaia data processing (Mignard et al., 2007).

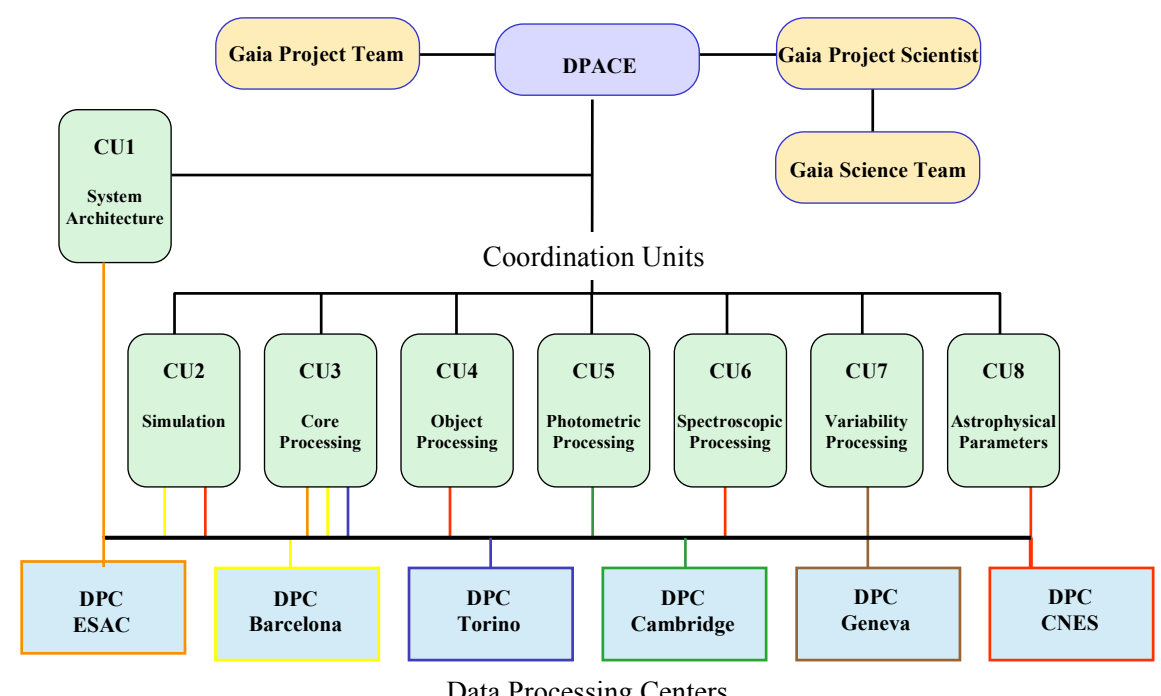

Figure 2. Top level structure of the DPAC with the Coordination Units (CUs) and the data processing centers (DPCs). Colored connectors indicate the link between the CUs and the DPCs.

The Consortium is structured around a set of eight Coordination Units (CUs) each in charge of a specific aspect of the data processing. The CUs are the building blocks of the Gaia Data Processing and Analysis Consortium and they are listed together with their leader's name in Table 1. An additional CU (the CU9) in charge of developing the tools for the Catalogue access by the scientific community is planned in the near future but is not yet activated. The CUs have clearly-defined responsibilities and interfaces, and their boundaries match naturally with the main relationships between tasks and the associated data flow. Responsibilities of the coordination units include: (a) defining data processing tasks and assigning responsibilities; (b) establishing development priorities; (c) optimizing, testing and implementing algorithms; (d) verifying the quality of the science products. Each coordination unit is headed by a scientific manager (the CU leader) assisted by one or two deputies and, where appropriate, a technical manager. The management team of each $\mathrm{CU}$ is responsible for acquiring and managing the resources 
needed for their activities. While the CUs are primarily structured for software development, all of them are closely associated with at least one DPC where the algorithms will be executed for the data processing in the operational phases.

The Consortium is coordinated by the 'Data Processing and Analysis Consortium Executive' (DPACE) committee. This top-level management structure deals with matters that are not specific to the internal management of a $\mathrm{CU}$, defining standards and policies needed to ensure an efficient interaction between all the CUs. Consistent with the Science Management Plan, the DPACE and its chair will serve as an interface between the DPAC and the Project Scientist and the Gaia Science Team. They are ultimately responsible for the data processing carried out by the DPAC. This executive committee is composed at the moment of the DPAC chair and deputy, the leaders of each CU, a representative of the CNES Data Processing Center. The Gaia Project Scientist (an ESA position) has a standing invitation to the DPACE where he has the status of observer.

Table 2. The Data Processing Centres associated to the DPAC, their current manager and involvement.

\begin{tabular}{clll}
\hline DPC & Location & Manager & Linked to: \\
\hline DPC-B & Barcelona & S. Girona & CU2, CU3 \\
DPC-C & CNES & X. Passot & CU2, CU4, CU6, CU8 \\
DPC-E & ESAC & J. Hoar & CU1, CU3 \\
DPC-G & Geneva & M. Beck & CU7 \\
DPC-I & Cambridge & F. van Leeuwen & CU5 \\
DPC-T & Torino & A. Volpicelli & CU3 \\
\hline
\end{tabular}

The DPAC has responded to the Announcement of Opportunity released by ESA on 9 November 2006. The Response document contains, in an hefty volume of more than 650 pages, the overall description of the Gaia data processing to reach the scientific objectives, the organization of the consortium and the funding commitments of the national agencies supporting the DPAC. This response has been reviewed by various ESA advisory committees and after one iteration the DPAC proposal and its selection have been formally endorsed by the ESA Science Program Committee in its meeting of May 2007. In November 2007 the same high level committee has also approved the funding agreement between the national agencies and ESA.

\section{Acknowledgements}

This short review of the DPAC organization and activities relies heavily on the contribution of many members of the DPAC who are collectively gratefully acknowledged for their dedication to the project.

\section{References}

FAH, 2007, http://folding.stanford.edu/

Lammers, U., 2006, Agis technical report, GAIA-C1-PR-ESAC-UL-018-1.

Lindegren, L., et al., 2008, this volume p.217

Mignard, F., et al., 2007, Proposal for the Gaia Data Processing, GAIA-CD-SP-DPAC-FM-0302, Mignard, F., Drimmel, eds.

Perryman, M., 2004, Estimation of Gaia Data Processing FLOPs, GAIA-MP-009. 